21.1

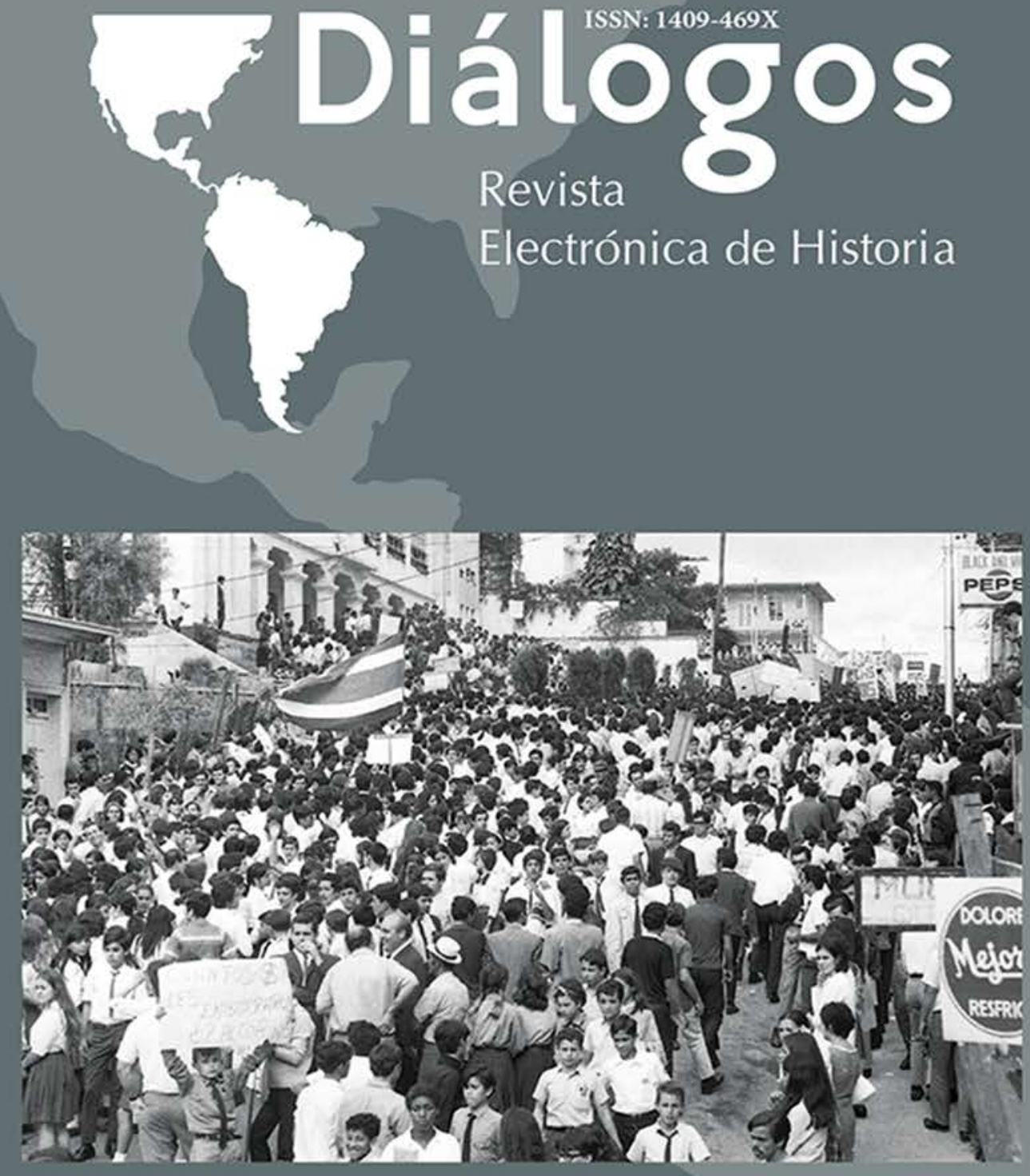

Centro de Investigaciones Históricas de América Central. Universidad de Costa Rica

\title{
Enero-junio 2020
}

url: http://revistas.ucr.ac.cr/index.php/dialogos/index

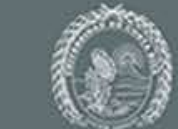

UNIVERSIDADDE

COSTARICA

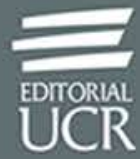




\title{
COLABORACIONES ACADÉMICAS Y BALANCE GENERAL DE LA PRODUCCIÓN HISTORIOGRÁFICA EN TEMAS DE CIENCIA, TECNOLOGÍA Y MEDIO AMBIENTE EN COSTA RICA
}

\author{
David Chavarría Camacho
}

\begin{abstract}
Resumen
Este artículo realiza una caracterización y sistematización general de las principales investigaciones académicas que tratan sobre los procesos históricos y su relación con el surgimiento y desarrollo de los fenómenos en ciencia, tecnología y medio ambiente en Costa Rica. Se determinan las principales problemáticas que analizan dichos autores, así como las temáticas generales de sus investigaciones y algunos vínculos y colaboraciones académicas entre ellos, lo que permite acercarse a un diagnóstico general del estado del conocimiento en estos campos de estudio. Para realizar este artículo, se emplearon métodos gráficos para analizar las asociaciones entre los investigadores, lo que permite determinar los componentes más generales de la estructura intelectual de los participantes de esta producción académica. La investigación considera tres componentes principales en dicho proceso: 1) los trabajos que tratan la historia de la institucionalización de las disciplinas científicas; 2) aquellos que llevan a cabo un análisis histórico de las relaciones entre el ser humano y la naturaleza en Costa Rica y, vinculado a este, 3) los trabajos que analizan la técnica, la tecnología y el medio ambiente en la historia agraria costarricense.

Palabras clave: Historia, ciencia, tecnología, medio ambiente, ciencia y sociedad, historia de las ciencias, análisis historico, análisis comparativo, análisis de la información
\end{abstract}




\title{
ACADEMIC COLLABORATIONS AND GENERAL BALANCE OF HISTORIOGRAPHIC PRODUCTION IN SCIENCE, TECHNOLOGY, AND ENVIRONMENT IN COSTA RICA
}

\begin{abstract}
This article realizes a general characterization and systematization of the main academic research that deals with historical processes and their relationship with the emergence and development of scientific, technological, environmental phenomena in Costa Rica. This paper discusses the main problems raised by these authors, the general themes of their research, and some academic links and collaborations between them, which allows us to approach a general diagnosis of the state of knowledge in these fields of study. To carry out this article, some graphic methods were used to analyze the associations between the researchers, which allows to determine the more general components of the intellectual structure of the participants of the academic production. The research considers three main components in this process: 1) the research studies that deals with the history of the institutionalization of scientific disciplines; 2) those who carry out a historical analysis of the relationship between human beings and nature in Costa Rica, and 3) the works that analyze technique, technology, and the environment in Costa Rican agrarian history.
\end{abstract}

Keywords: history, science, technology, environment, science and society, history of science, historical analysis, comparative analysis, information analysis 


\section{INTRODUCCIÓN}

Con el fin de generar una aproximación válida a las principales investigaciones académicas que tratan sobre los procesos sociales y su relación con el surgimiento y desarrollo de los fenómenos científicos, tecnológicos y ambientales en Costa Rica a lo largo del tiempo, este artículo realiza una caracterización y sistematización general de las publicaciones y proyectos de investigación afines. En primera instancia, se determinan las principales problemáticas que analizan diversos autores competentes, así como las temáticas generales de sus investigaciones y algunos vínculos y colaboraciones académicas, lo que permite comenzar a acercarse a un diagnóstico general del estado del conocimiento en estos campos de estudio.

Para el desarrollo de este artículo, se emplearon algunos métodos gráficos para analizar las asociaciones entre los investigadores, que permiten determinar los componentes más generales de la estructura intelectual de los participantes de la producción académica. Dentro del análisis de las relaciones entre los individuos, se incluyeron más de treinta autores relevantes para el periodo comprendido entre 1990 y 2015 , los cuales estudian temáticas relativas a la historia, la sociedad y sus múltiples vínculos con la ciencia, la tecnología y el medio ambiente.

\section{HISTORIA DE LA INSTITUCIONALIZACIÓN DE LAS DISCIPLINAS CIENTÍFICAS EN COSTA RICA}

En la Tabla 1, se presenta una caracterización básica de los estudios históricos de temas científicos que han sido investigados para el caso específico de la Universidad de Costa Rica. Allí se observan tres grandes problemas de investigación, que tratan aspectos relativos a la institucionalización de las disciplinas científicas. Dichas publicaciones se preocupan principalmente por estudiar cómo nacen y se desarrollan diversas disciplinas científicas tradicionales o clásicas. Dicho grupo de trabajos corresponden a los más antiguos y abundantes para el caso de Costa Rica. En la Figura 1, se muestra una distribución general de sus ejes temáticos y sus principales problemas de investigación. En esta, se pueden observar estas investigaciones concentradas a la derecha del Eje Y.

Los primeros trabajos académicos que abordan temáticas científicas datan de principios de la década de 1990, con varios textos -que ya podrían ser clásicos- del matemático costarricense Ángel Ruiz Zúñiga, que estudian, a nivel general, la historia de las matemáticas en Costa Rica y datan la construcción de la disciplina a través del escrutinio de diferentes fuentes escritas de primera mano, analizando para ello a los principales actores del proceso, los eventos políticos que determinaron su carácter, así como una historia comparada de la adopción de las ideas filosóficas y matemáticas emanadas desde los centros hegemónicos del conocimiento científico hacia Costa Rica (Ruiz, 1990, 1995, 2001; Barrantes \& Ruiz, 2000; Ruiz \& Rodríguez, 2003). 


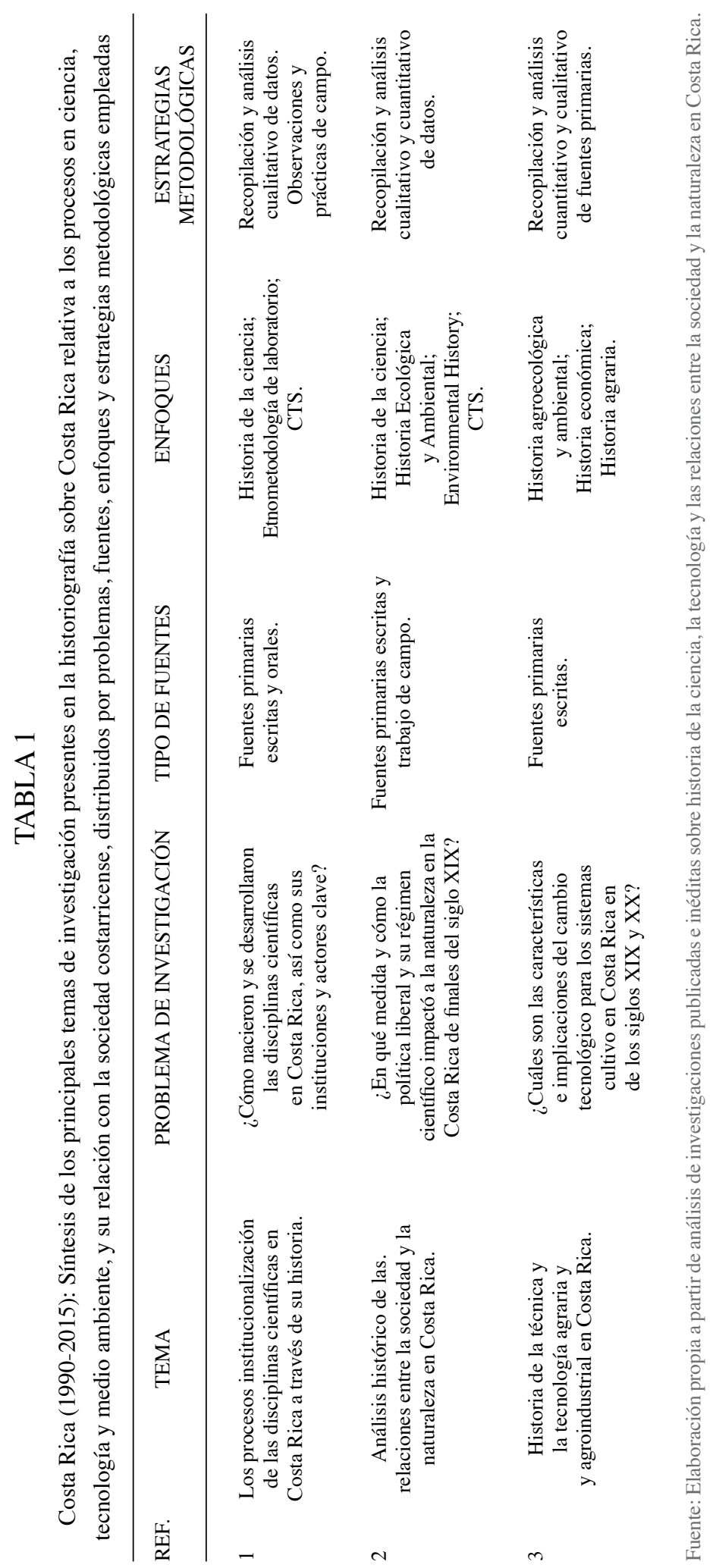




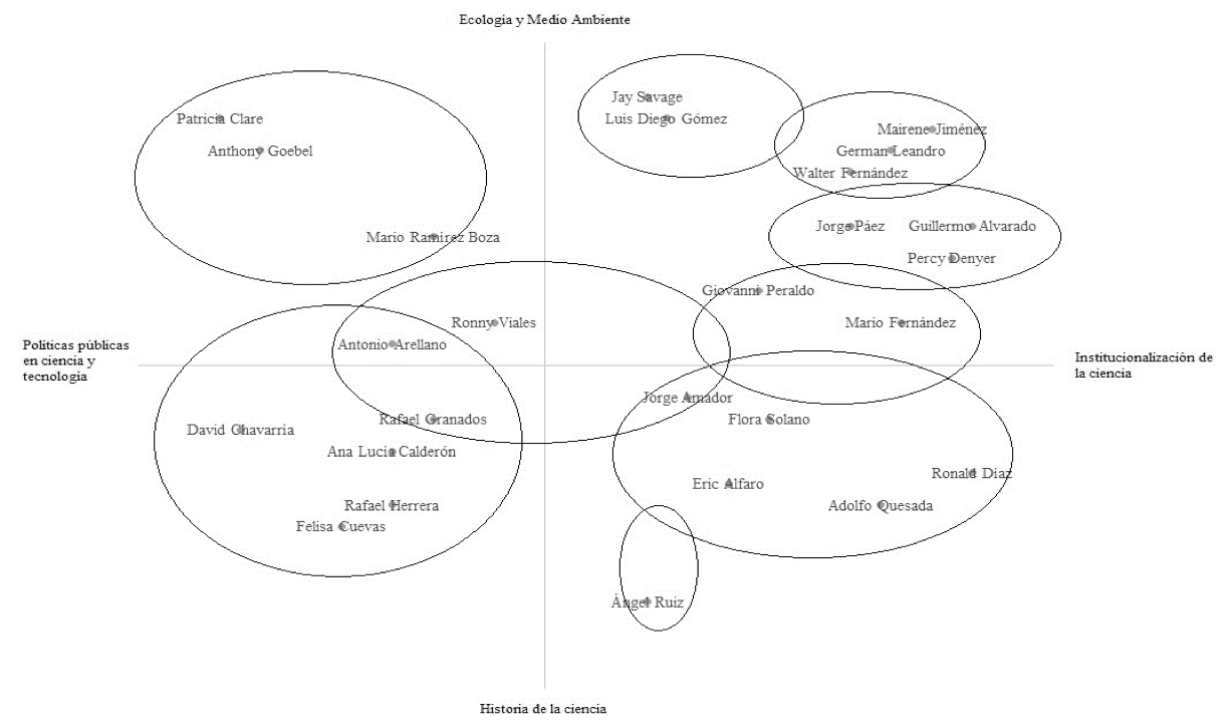

Figura 1. Análisis de los componentes principales (investigadores) en temas de ciencia, tecnología y medio ambiente y su relación con la sociedad ordenados según problemática y temática estudiada (1990-2015). Fuente: Elaboración propia a partir de análisis de las co-autorías en las publicaciones de los autores relativas a temas de ciencia, tecnología y medio ambiente, y su relación con los procesos sociales (1990 y 2015).

Como se puede observar a partir del estudio de sus publicaciones, Ruiz Zúñiga trabajó dichos temas con base en el establecimiento de vínculos colaborativos con una serie de investigadores afines. Lo que llama la atención de estas publicaciones es que no solamente se concentra en la buena elaboración de sus argumentos, su capacidad analítica o su uso riguroso de las fuentes, sino el hecho de que ya desde este primer aporte en el campo -una recopilación titulada Las Matemáticas en Costa Rica (1990)-, Ruiz Zúñiga fue capaz de establecer nexos con una serie de investigadores que en ese entonces comenzaban a incursionar en el campo de la historia social de la ciencia en el país. Llama especialmente la atención el vínculo que establece el matemático con tres investigadores que, para 1990, se encontraban ligados al Centro de Investigaciones Geofísicas (CIGEFI) de la Universidad de Costa Rica (UCR). Se trata del físico Jorge Amador Astúa, la historiadora de la ciencia Flora Solano Chaves y el astrofísico Jorge Páez Portuguez, quienes colaboraron en dicha compilación con un artículo titulado Algunos detalles y hechos históricos de los albores de la Física en Costa Rica (en Ruiz, 1990), presentado durante el Tercer Congreso Nacional de Matemáticas, que se llevó a cabo en octubre de ese año en la UCR y cuyo organizador principal fue Ruiz, quien además presidía la Asociación Matemática Costarricense (AMC), institución encargada del evento.

Dentro de esta serie de trabajos pioneros, se puede rescatar también el de Luis Diego Gómez en conjunto con el biólogo estadounidense Jay M. Savage (1991), titulado Investigadores en aquella rica costa: Biología de campo costarricense (1400-1980), en donde se incursiona en el rescate histórico de los estudios biológicos llevados a cabo en el país a lo largo de los siglos. Otro de los estudios importantes en este sentido, corresponde al trabajo Historia del campo geomagnético en Costa Rica, 
publicado por Páez, Jiménez y Leandro (1995), en donde realizan un rescate histórico de los científicos decimonónicos Sir Edward Belcher (1836) y Henry Pittier (1898), a través de las observaciones sistemáticas del campo magnético del país durante el siglo XIX y principios del XX. Otro de los trabajos importantes fue presentado por Páez (1994) y se tituló Aspectos históricos de la Astronomía en Costa Rica.

Estas investigaciones, además de muchas otras que fueron publicadas durante esa década, dan cuenta del surgimiento de un nuevo campo de estudio en el país y de la necesidad que existía en estos círculos académicos por el rescate de problemas relativos a la producción científica desde una perspectiva histórica, problema de investigación que había sido dejado de lado, hasta ese momento, por la historiografía nacional. Este conjunto de investigaciones abrió, en ese momento, la posibilidad de comprender por qué y cómo se han desarrollado las ideas y productos científicos en Costa Rica durante el último siglo, la cuales fueron posibles a través del establecimiento de empresas e instituciones de carácter privado y estatal, impulsadas por científicos, empresarios y políticos.

Por otro lado, la aplicación de un enfoque histórico al estudio de cada uno de sus casos les permitió plantear y resolver problemas de investigación relativos al estímulo de la ciencia y la tecnología como elementos clave para el fortalecimiento de las ideas de progreso prevalecientes en Costa Rica desde finales del siglo XIX.

Un análisis detenido de la producción académica del CIGEFI durante su existencia (Calvo \& Alfaro, 2018), evidencia el trabajo llevado a cabo sobre este tipo de problemáticas. Trabajo impulsado principalmente por Amador y Solano. Entre 1990 y 2002, se llevaron a cabo una serie de investigaciones cuyos ejes de analíticos principales consistieron en el estudio de la institucionalización de la física, la meteorología, las ciencias atmosféricas en general, las ciencias del espacio, así como los significados sociales de algunos fenómenos astrales como los eclipses de Sol que fueron observados en Costa Rica durante el siglo XIX (Solano \& Páez, 1990; Solano \& Amador, 1991; Amador, Páez \& Solano, 1990; Páez, 1994; Solano \& Amador, 1994; Fernández, 1994; Amador citado en Peraldo, 2002b; Amador, 1997, 2002a).

A estos esfuerzos académicos por el rescate de la historia de la ciencia en el país, se unieron, durante la década de 1990, el físico atmosférico Walter Fernández Rojas (1995, 1998, 2000) (Fernández \& Páez, 2000), y el geólogo Giovanni Peraldo Huertas (2002) (Peraldo \& Amador, 2007; Peraldo, Solano \& Quesada, 2009), quienes comenzaron a desarrollar a partir de una base analítica descriptiva y con un uso extensivo de fuentes escritas de primera mano diversos temas en la historia de las instituciones científicas de Costa Rica y sus principales gestores, empresas generalmente ligadas al estudio de los fenómenos climáticos, atmosféricos, astronómicos, geológicos y de las ciencias médicas en Costa Rica durante los siglos XIX y XX. A esta corriente de investigaciones se unió finalmente el historiador Ronald Díaz Bolaños, quien hasta la actualidad ha realizado una serie de estudios en este ámbito, en conjunto con Solano (Díaz, 2003, 2005, 2007, 2009; Díaz \& Solano, 2006; Viales, Amador \& Solano, 2009).

Todas estas iniciativas fueron estimuladas por medio de una serie de proyectos de investigación y actividades académicas y de acción social (Viales, 2003), que 
posibilitaron aún más la consolidación de un campo de estudio a nivel institucional y permitieron el establecimiento de las primeras redes de investigación en el extranjero. Dos de las tareas más importantes de estos académicos fueron el proyecto denominado Meteorología e impacto social regional: Centro América y México (MISCAM), apoyado por la Vicerrectoría de Investigación de la UCR e inscrito en el CIGEFI en 1997, el cual contó con la participación de Amador, Bolaños, el historiador Francisco Enríquez Solano y la geógrafa Adriana Bonilla Vargas. Sus objetivos generales consistían en estudiar por medio de la relación existente entre diferentes acontecimientos históricos y científicos, el desarrollo de la meteorología en la región y contribuir al conocimiento sobre el impacto de esta disciplina en las sociedades centroamericanas y en México (Vicerrectoría de Investigación, 1997).

Este proyecto permitió, entre otras cosas, la presentación de una mesa de trabajo titulada Historia y Entorno Natural, durante el VI Congreso Centroamericano de Historia llevado a cabo en Panamá en el 2002, que fue uno de los primeros espacios que se abrieron para que los investigadores centroamericanos pudieran discutir temas relativos a la historia de las ciencias naturales en la región y el papel de los historiadores en este campo, discusión que quedó materializada en el artículo La función del historiador en los estudios climáticos aportes y perspectivas, presentado por Enríquez (2001). También promovió la publicación de otras investigaciones relativas al tema del clima y el cambio climático (Amador, 2002a; Amador citado en Peraldo, 2002b).

Todas estas investigaciones reseñadas en los párrafos anteriores fueron realizadas por investigadores provenientes de diferentes disciplinas de las ciencias naturales y de las ciencias sociales, principalmente historiadores. Esta fue, quizás, la característica principal de uno de los programas más importantes para el estudio histórico y social de la ciencia y la tecnología en Costa Rica. Se trata del Programa de Estudios Sociales de la Ciencia, la Técnica y el Medio Ambiente (PESCTMA), que se estableció en el CIGEFI a partir del año 2003, al cual se hará referencia en las siguientes secciones. El establecimiento de este programa permitió la incorporación continua de investigadores que trabajaron diversos temas desde una perspectiva multi, trans e interdisciplinaria.

A modo de balance general sobre la historia de la institucionalización de las disciplinas científicas en Costa Rica, se puede señalar tres aspectos fundamentales dentro del desarrollo de la historiografía costarricense. En primera instancia, se lleva a cabo un rescate intelectual de los procesos históricos referentes al desarrollo de la ciencia en Costa Rica desde finales del periodo colonial, tema que había sido tratado de manera marginal en los estudios históricos existentes. Segundo, el acercamiento a estos temas creó un vínculo colaborativo con diversos académicos de otras disciplinas de las ciencias naturales que estaban interesados en investigar la trayectoria histórica de sus disciplinas de base, logrando con esto los primeros pasos para una integración interdisciplinaria en lo que respecta al estudio socio-histórico de la ciencia y la tecnología. Tercero, permitió comenzar a trazar lineamientos para la formación e incorporación de nuevos investigadores en el campo, permitiendo establecer vínculos a nivel regional con otros investigadores preocupados por este tema de investigación. 
Finalmente, en lo que respecta a los alcances reales a la historia de la institucionalización de las disciplinas científicas en Costa Rica, se podría decir que esta comunidad precursora logró una primera aproximación a nuevos problemas de investigación para la historiografía costarricense y centroamericana. Sin embargo, ha carecido hasta la fecha de herramientas teóricas y metodológicas adecuadas para el análisis de los fenómenos sociotécnicos y tecnocientíficos, quedándose, en la mayoría de los casos, en reconstrucciones históricas de corte más bien clásico, desprovisto de acercamientos claros a las tendencias mundiales en historia de la ciencia y la tecnología y de tendencias analíticas mucho más elaboradas, como las que se realizan desde los estudios en Ciencia, Tecnología y Sociedad (CTS), la sociología de la tecnología y otras disciplinas afines. Las últimas perspectivas, como se verá a continuación, comenzaron a incorporarse a partir del 2003 con la creación del PESCTMA.

\section{ANÁLISIS HISTÓRICO DE LAS RELACIONES ENTRE EL SER HUMANO Y LA NATURALEZA EN COSTA RICA}

Una vez instituido el PESCTMA, se desarrolló paralelamente un componente ecológico y medio ambiental ligado al análisis histórico de las relaciones entre el ser humano y la naturaleza, que se nutrió principalmente por los aportes dados en este campo por los historiadores Ronny Viales, Patricia Clare, Anthony Goebel y Mario Ramírez. En la Figura 2, se observan las relaciones colaborativas de Viales con los demás investigadores a los que se ha hecho referencia en la sección anterior.

\footnotetext{
1.Ángel Ruiz Zúñiga 2.Jorge Páez Portuguez 3.Jorge Amador Astúa 4.Flora Solano Chaves 5.Luis Diego Gómez 6.Jay M. Savage 7.Mairene Jiménez García 8. Guillermo Alvarado Induni 9.Walter Fernández Rojas 10. Giovanni Peraldo Huertas 11.Mario Fernández Arce 12. Ronald Díaz Bolaños 13.Anthony Goebel Mc Dermott 14.Patricia Clare Rhoades

15.Ronny Viales Hurtado 16.Rafael Granados Carvajal 17.Eric Alfaro Martínez 18. Rafael Herrera González 19.Felisa Cuevas Cordero 20.Mario Ramírez Boza

21.Antonio Arellano Hernández 22. Ana Calderón Saravia 23.David Chavarría Camacho 24.Adolfo Quesada Román
}
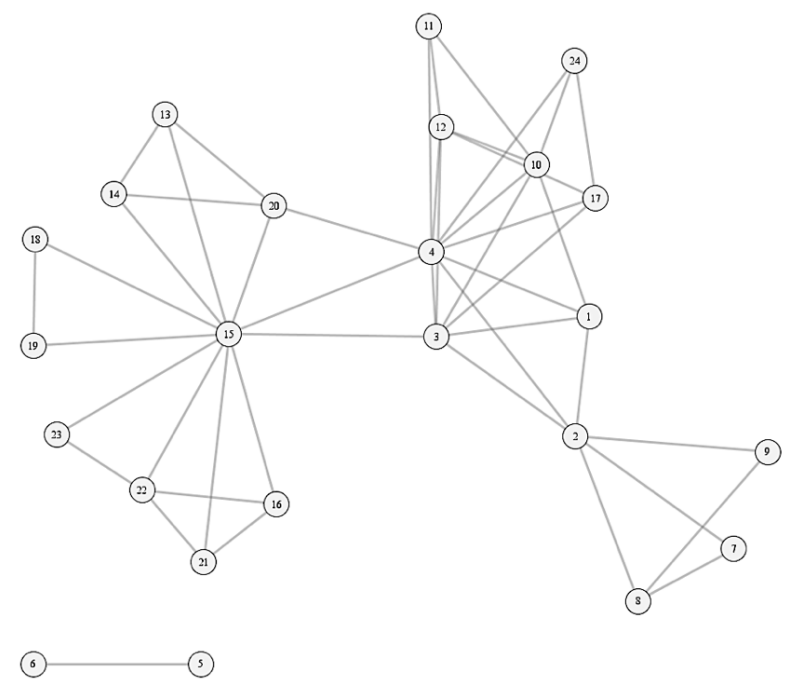

Figura 2. Relaciones entre investigadores universitarios que han desarrollado temas relativos a la ciencia y la tecnología en su dimensión histórica y social (1990-2015). Fuente: Elaboración propia a partir de análisis de las publicaciones del Centro de Investigaciones Geofísicas de la Universidad de Costa Rica entre 1979 y 2015. 
En esta ilustración, se puede advertir a Viales como un actor clave en la articulación de las diferentes ramas temáticas llevadas a cabo por el PESCTMA. Esto se debe a que es el investigador que presenta la mayor cantidad de vínculos, representados por las líneas o aristas que unen a cada uno de los actores, también llamados nodos. Del grafo se desprenden, al lado derecho de la imagen, un grupo importante de investigadores definido a partir de sus problemas de investigación. Esta comunidad la constituyen Viales, Goebel y Clare, quienes además logran converger con el primer gran grupo de estudiosos de los procesos históricos de institucionalización de las ciencias en Costa Rica.

Esta convergencia se llevó a cabo a través de diversas investigaciones desarrolladas en conjunto. La principal y gran labor colaborativa se materializó en el libro Concepciones y representaciones de la naturaleza y la ciencia en América Latina, editado por Viales, Amador y Solano (2009), fundadores del PESCTMA. Este texto integró un estudio general de las representaciones de la ciencia en Costa Rica y América Latina, así como de los vínculos existentes entre la naturaleza, el régimen liberal y sus proyectos tecnocientíficos de reorganización productiva de la naturaleza en Costa Rica hacia finales del siglo XIX.

Dos de las investigaciones que permiten establecer un vínculo problemático y metodológico directo entre la historia ambiental y la historia de la ciencia y sus instituciones -descritos en la sección anterior-, son El Estado, lo transnacional y la construcción de comunidades científicas en la Costa Rica liberal (1870-1930): La construcción de un régimen de cientificidad, de Viales y Clare; y Naturaleza, mercado y progreso: las justificaciones de la reorganización productiva de la naturaleza en los exploradores extranjeros en la Costa Rica decimonónica (1850-1905) de Goebel. Ambos contenidos en la compilación de Viales, Amador y Solano (2009). El primero de ellos se dedica a "analizar los procesos sociales, políticos, económicos y culturales que contribuyeron a conformar una política científica en la Costa Rica de finales del siglo XIX y principios del XX", y el segundo se encarga del "análisis del contenido de los escritos de varios naturalistas que visitaron Costa Rica a finales del siglo XIX e inicios del XX" (Viales, Amador \& Solano, 2009, p. 1), con el fin de determinar a través de estas fuentes las concepciones y representaciones que tenían los liberales y los científicos acerca de la naturaleza, vista como un objeto ocioso que debía ser transformado por el ser humano (Goebel, 2008, 2009, 2013; Goebel \& Viales, 2010).

Las investigaciones de este tipo se ubican en el cuadrante superior izquierdo de la Figura 1 y permiten dilucidar el carácter histórico de distintas ramas del conocimiento en ciencias naturales a través del trabajo conjunto con los científicos preocupados por los análisis de trayectoria. Por medio de la historia ambiental, también se establece un vínculo entre los investigadores del CIGEFI y los historiadores del Centro de Investigaciones Históricas de América Central (CIHAC), los cuales investigaron de manera continua en ambos centros de investigación durante una década. 
Viales rescata que en el CIHAC, desde el 2002 y a través del Programa de Investigación en Historia Económica y Social, se habían venido discutiendo y publicando trabajos relativos al tema dentro del grupo de discusión en historia ecológica y ambiental y el grupo de discusión en historia social de las ciencias médicas y la salud pública, cuyo mayores aportes fueron presentados en los últimos años por la historiadora Ana María Botey Sobrado (2013, 2019), con su tesis doctoral en historia titulada Los actores sociales y la construcción de las políticas de salud del Estado liberal en Costa Rica (1850-1940), de la cual ha derivado la que es quizás la publicación más relevante en historia de la salud pública en Costa Rica: Los orígenes del Estado del Bienestar en Costa Rica: salud y protección social (1850-1940), obra publicada en 2019. Estas investigaciones recogen todos los resultados que durante más de diez años se han llevado a cabo en el campo de la historia de la salud pública y la institucionalización de la medicina en Costa Rica.

Por otro lado, esta comunidad ha venido realizando estudios sobre el diseño de políticas públicas en la Costa Rica contemporánea, que han permitido evidenciar cómo la implementación y el desarrollo de diversos proyectos en ciencia y la tecnología tienen implicaciones sobre la sociedad costarricense al excluirse de los beneficios a una gran parte de la población. Es por esta razón que abogan por un estímulo a los procesos socio-técnicos de toma de decisiones que posibiliten la cohesión social no solo en Costa Rica sino en América Latina en general (Viales, 2010). Así, su problema de investigación ha consistido en estudiar en qué medida y cómo las políticas en ciencia y tecnología pueden colaborar en los procesos de cohesión social, señalando que la desigualdad, la exclusión y la discriminación que acarrea el desarrollo de nuevas tecnologías tienen respuesta y deben necesariamente estudiarse desde una perspectiva histórica. Esto quiere decir que el fenómeno científico y tecnológico debe comprenderse a través del tiempo y en su dimensión espacial, es decir, en el papel que cumple la región dentro del entorno global.

El balance general de estos trabajos permite identificar la existencia de un vínculo mucho más estrecho entre los estudios en perspectiva histórica de las instituciones científicas con investigadores formados en el campo de la historia, los cuales introdujeron nuevos problemas y métodos de investigación, y comenzaron a establecer vínculos académicos internacionales que se mantienen hasta la actualidad, entre los cuales se puede mencionar los ligámenes directos con la Asociación Latinoamericana de los Estudios Sociales de la Ciencia y la Tecnología (ESOCITE), la Society for Social Studies of Science (4S), el Programa Iberoamericano de Ciencia y Tecnología para el Desarrollo (CYTED) y la Red Mexicana de Estudios Sociales de la Ciencia, la Tecnología y la Innovación (RESoCTI). Lo anterior evidencia la incorporación del país dentro de los círculos más importantes de investigación a nivel mundial relativos a los fenómenos sociales en ciencia, tecnología y medio ambiente, permitiendo ampliar el cuerpo teórico y metodológico de conocimiento local, la incorporación de nuevos temas y problemas de investigación y de nuevos investigadores. 
En este contexto, uno de los aportes de mayor relevancia en la historiografía costarricense llevado a cabo por esta comunidad ha sido la combinación de problemáticas propias de los estudios sociales de la ciencia y la tecnología con la relación histórica entre el ser humano y la naturaleza. Esta ha confluido en la actualidad en la generación de nuevos estudios desde una perspectiva socio-tecno-ambiental, a partir de la creación de un nuevo programa de investigación en el CIHAC, denominado Ambiente, ciencia, tecnología y sociedad (ACTS). Intersección Entre historia ambiental y estudios sociales de la ciencia, la tecnología y la sociedad(CTS) que entró en vigencia en el 2016 (Vicerrectoría de Investigación, 2016). El objetivo general de este programa ha sido el de desarrollar confluencias o interacciones entre los enfoques teóricos de la historia ambiental y de los estudios sociales de la ciencia y la tecnología, logrando una participación activa y organización de actividades académicas nacionales e internacionales. En términos cualitativos, los trabajos expuestos y publicados por el programa ACTS han tendido a reunir perspectivas tanto de la filosofía, la sociología, la antropología y la historia, lo cual demuestra el sostenido esfuerzo interdisciplinario de los integrantes del Programa. Sus investigadores e investigadoras, además, han abordado problemas de estudio en el contexto global de la historia socio-ambiental, técnica y científica, y desde el enfoque comparado tanto regional como local. El Programa también se ha caracterizado por posicionar sus resultados en actividades académicas y productos internacionales, integrando a investigadores extranjeros. Tres investigadores incorporados a las actividades de este Programa son la Dra. Rosalba Casas Guerrero (México), el Dr. Gabriel Matharán (Argentina) y el Dr. Juan Pablo Zabala (Argentina), especialistas en diferentes campos de la historia de la ciencia y los estudios sociales de la ciencia y la tecnología, quienes actualmente participan en un proyecto de creación de una red temática de historia global de la microbiología (Vicerrectoría de Investigación, 2018).

Desde la perspectiva de la historia ecológica y ambiental hay grandes aportes de los investigadores de la Universidad Nacional (UNA), quienes han estimulado desde hace varias décadas la apertura de nuevas áreas de investigación en los campos de la historia ecológica y ambiental. Entre los estudios recientes desde este enfoque, se pueden señalar los trabajos de Yanina Pizarro Méndez y Jorge Marchena Sanabria (2016a,2016b) sobre cuencas hidrográficas y acceso al agua en Guanacaste; las investigaciones de Maximiliano López López y Roberto Granados Porras (2016) y Rafael Ledezma Díaz (Ledezma \& Granados, 2008; Granados, 2013) dedicadas al análisis del modelo de conservación, los parques nacionales, la explotación de maderas y la producción de carbón; la investigación de Yendry Vargas Trejos (2015) sobre la exposición de trabajadores agrícolas a insumos químicos, así como los aportes de Wainer Coto Cedeño $(2015,2016)$ sobre sistemas agroalimentarios y producción de papa. Otros trabajos anteriores de investigadores de la UNA que cabe destacar son los de Emilio Vargas Mena $(2000,2001)$ sobre educación ambiental y territorios indígenas y la vasta producción de Wilson Picado Umaña $(2011,2012,2013)$ sobre Revolución Verde y otras investigaciones en historia ecológica y agro-ambiental a las cuales se hará referencia en el próximo apartado. 


\section{LA TÉCNICA, LA TECNOLOGÍA Y EL MEDIO AMBIENTE EN LA HISTORIA AGRARIA COSTARRICENSE}

Otro de los grandes grupos afines al tema de investigación que convoca este balance general corresponde a los estudios relativos y la tecnología agraria y agroindustria en Costa Rica. Este campo de investigación ha sido liderado por los investigadores de la UNA y han publicado sus trabajos mayoritariamente en la Revista de Historia. ${ }^{1}$ Esta proviene de campos de estudio ligados a la historia económica, la historia agraria y agroindustrial, los cuales poseen vínculos colaborativos débiles con la comunidad anteriormente descrita.

Estas investigaciones tienen la característica de que no están ligadas al paradigma de los estudios en Ciencia, Tecnología y Sociedad (CTS) como sí los están una gran cantidad de trabajos descritos en la sección anterior. Estos parten más bien de una reconstrucción y un análisis sobre la historiografía agraria de Costa Rica que les permite visibilizar, basados en diversas fuentes escritas de primera mano, el proceso histórico de cambio tecnológico de las actividades agrícolas. Este grupo de investigaciones parten de varios estudios realizados en el extranjero por Charles Loomis, Morales, Clifford y Olen (1958), Antonio Arce (1959) y por otros trabajos pioneros llevados a cabo por la geógrafa e historiadora Carolyn Hall (1978) y el historiador José Antonio Fernández (1986).

El principal investigador del cambio tecnológico en el campo es el historiador Mario Samper Kutschbach con los textos Policultivo, modernización y crisis: paradojas del cambio técnico/social en la caficultura centroamericana (1993) y Cambio tecnológico, ciclo laboral y productividad del trabajo en una empresa cafetalera costarricense, 1946-1961 (1994), trabajo publicado en 1994 en conjunto con Margarita Rojas y Margarita Torres. En el último se estudia el proceso de inserción de las nuevas técnicas agrícolas establecidas a mediados del siglo XX con el fin de aumentar los rendimientos en la producción agrícola en el contexto favorable de la posguerra, que permitió la intensificación de la caficultura mediante la extensión de los cultivos. Analiza, finalmente, los cambios en la fuerza de trabajo, su organización con respecto a la producción total y cómo esta última se vio afectada, o no, por la introducción de nuevas tecnologías. Esto lo hace a través del estudio de la organización técnica del trabajo (véase también Aguilar, Barboza \& León, 1982; Cléves, 1995; Rojas, 2000; Samper, 2001). Samper Kutschbach realiza así una tipología económica del impacto de la modernización de distintas fincas productoras de café en Centroamérica, lo cual abre estas posibilidades de estudios de la tecnología rural en el caso costarricense y centroamericano (véase también Samper 1994; Samper, Naranjo \& Sfez, 2000). Cabe mencionar en este punto, el gran trabajo de Paul Sfez (2000) titulado Revolución Verde y cambio tecnológico en la caficultura, en donde se recontruyen los cambios tecnológicos del café en el contexto de la Revolución Verde. 
A nivel general, la historiografía sobre el cambio tecnológico en la caficultura costarricense se ha preocupado por determinar el impacto de la tecnología en los procesos productivos y en las características de la fuerza laboral, distinguiendo, temporalmente, la evolución y las características de cada uno de los procesos de producción. Samper (1993) evidencia muy bien esta idea desde sus primeros artículos sobre el tema, en donde manifiesta la necesidad de observar cómo la introducción de la tecnología afectó a los sistemas sociales de producción y viceversa, cómo la distribución social del trabajo impactó y condicionó la introducción de nuevas tecnologías agrícolas. Finalmente, Samper va más allá y realiza un análisis de cómo estas transformaciones socio-técnicas estuvieron condicionadas por los distintos cambios en el mercado internacional del café.

Siguiendo esta misma línea de trabajo, otro de los autores que han estudiado el cambio técnico en la caficultura con mayor detalle es Carlos Naranjo Gutiérrez (2007), con su tesis de Maestría titulada La modernización de la caficultura costarricense 1890-1950, y otra publicación realizada en conjunto con Samper y Paul Sfez (2000). El otro investigador importante en este grupo de estudiosos es Wilson Picado (Picado, 2000; Picado, Ledezma \& Granados, 2009; López \& Picado, 2012), quien en los últimos años ha presentado una serie de trabajos relativos a la tecnología empleada en la caficultura costarricense. Estos autores han estado ligados a los grupos de investigación de la UNA.

En el campo específico de la tecnología agrícola y el sector agroindustrial de la caña y otros productos, cabe destacar los aportes de Andrea Montero Mora, y los economistas Jorge León Sáenz y Nelson Arroyo Blanco (León \& Arroyo, 2012; León, Arroyo \& Montero, 2016). En esta misma tendencia, Montero Mora, historiadora e investigadora ligada actualmente al PESCTMA, ACTS y CIHAC, ha trabajo recientemente en conjunto con Viales en una investigación titulada Los inicios frustrados de la mecanización de la agricultura costarricense entre 1900 y 1950. La difusión del arado y del tractor. Actores, tecnología agrícola, discursos y representaciones desde una perspectiva transnacional (s.f). Esta última investigación rescata la trayectoria de la mecanización de la agricultura costarricense, que fue estimulada por las políticas agrarias liberales para introducir nuevos arados y tractores con el fin de llevar a cabo dichas tareas productivas. La invasión del campo por la tecnología en pro de la idea del progreso y riqueza de la nación, según su interpretación, se lograría a través del empleo de estos artefactos revolucionarios. Esta investigación, a diferencia de las anteriores, se realiza desde enfoques propios de los estudios CTS, vistos desde un punto de vista histórico y con un uso cualitativo de fuentes escritas.

Desde esta perspectiva CTS, se hace mención especial al trabajo de Patricia Clare (2011) titulado Los cambios en la cadena de producción de la palma aceitera en el Pacífico costarricense, en donde a partir de la confluencia de la historia económica, agroecológica y ambiental, así como de los estudios sociales de la ciencia y la tecnología, comprende los procesos de producción y generación de conocimientos relativos a la palma aceitera en la región del Pacífico Sur durante la segunda mitad del siglo XX. 
Este trabajo constituye un precedente para el análisis metodológico y teórico de las comunidades científicas desde el punto de vista histórico. Este tema se desarrolla principalmente en su primer capítulo titulado Reorganizaciones de los departamentos de investigación tecno-científica y cambios en los sistemas de cultivo de palma aceitera (1950-2007) (pp. 39-90).

El balance general de los trabajos relativos a la técnica y la tecnología en Costa Rica arroja la existencia de dos comunidades petenecientes a dos instituciones de investigación distintas. En primera instancia, una gran comunidad que ha estudiado rigurosamente el cambio tecnológico en la agroindustria que trabajan desde la Escuela de Historia y la Maestría en Historia Aplicada, ambas de la UNA, y que ha realizado sus interpretaciones a partir de reconstrucciones históricas del agro, utilizando todo el cuerpo de conocimiento y las herramientas desarrolladas al interior de su comunidad. A diferencia de la producción realizada en los programas de investigación desarrollados tanto en el CIGEFI como en el CIHAC, los trabajos realizados en la UNA no están directamente vinculados con la comunidad dedicada a los estudios sociales de la ciencia y la tecnología (CTS), direccionando sus esfuerzos en el análisis del componente tecnológico basada en su consolidada producción historiográfica en el campo de la historia agraria y agroindustrial y actualmente en la historia ecológica y ambiental.

En este sentido, la comunidad de historiadores de la UNA han observado en los últimos años un gran potencial derivado del estudio histórico del medio ambiente y sus múltiples vínculos con el agro costarricense, realizando, desde esta perspectiva, fuertes acercamientos a los trabajos publicados desde la historia ambiental noratlántica -la llamada environmental history- y la historia ambiental latinoamericana, en donde han tenido un fuerte impacto dentro de la Sociedad Latinoamericana y Caribeña de Historia Ambiental (SOLCHA), siendo los anfitriones del IX Simposio SOLCHA en el 2018.

A través de estos esfuerzos han logrado generar vínculos colaborativos con diversos especialistas de América Latina y España, ligados a dicha asociación. En esta última corriente, destacan los trabajos publicados por los historiadores Wilson Picado, investigador de la UNA, y Gloria Guzmán y Juan Infante Amate, investigadores del Laboratorio de Historia de los Agro-ecosistemas de la Universidad Pablo de Olavide de España. Estos trabajos son de vital importancia debido a que conjugan el gran bagaje en los estudios históricos del agro costarricense con el enfoque del metabolismo social y los flujos de energía y materiales, contando actualmente con metodologías para el análisis estadístico de datos a gran escala -mejor conocidos como big data- (Infante \& Picado, 2016, 2017; Infante, Picado \& Guzmán, 2017).

En el caso de los investigadores del CIHAC, estos también han incursionado en los últimos años en temas científicos y tecnológicos relativos a la historia agraria, como es el caso de Clare, Viales y Montero. Estos estudios tienen la particularidad de haber realizado acercamientos a las metodologías y problemas de investigación propios de los estudios en CTS, así como en el área de la environmental history y la historia ecológica y ambiental latinoamericanas, influidos igualmente por sus vínculos con la SOLCHA. 


\section{BALANCE GENERAL}

Ambas corrientes de la historia ecológica y ambiental, una originada en la Universidad de Costa Rica y otra originada en la Universidad Nacional, lograron confluir y materializarse en un proyecto en conjunto que se desarrolló entre 2010 y 2012, el cual recibió el apoyo de los fondos para la investigación FEES-CONARE que se obtuvieron gracias a la iniciativa y el empeño de los historiadores Juan José Marín Hernández y Carlos Hernández Rodríguez. Este mega-proyecto que llevó por título Tierra, aire, agua y bosques. Historia del medio ambiente en la Costa Rica de los siglos XIX y XX (Vicerrectoría de Investigación, 2010) convocó a diversos investigadores e investigadoras y consolidó una iniciativa reflexiva para construir una historia ambiental atinente a la realidad costarricense, construir una agenda común de investigación, e impulsar y proyectar el debate académico sobre los problemas teóricos y metodológicos inherentes al medio ambiente desde la perspectiva histórica. Esta iniciativa fue sin duda un gran paso en el proceso de construcción de este campo de conocimiento y estimuló la generación de vínculos investigativos entre diversas comunidades científicas.

De esta forma, la importancia de la revisión historiográfica realizada en estas páginas radica en que solamente a través del trazo de la trayectoria y de los vínculos existentes será posible una reconstrucción histórica integral y una visibilización clara de la producción de conocimiento sobre estos temas. Lo anterior permitirá integrar al conocimiento histórico previamente producido, una serie de nuevas preguntas y problemas de investigación relativos a los procesos de desarrollo científico, tecnológico y medioambiental que se ha llevado a cabo en el país al menos durante los últimos cincuenta años.

La conjugación del análisis histórico de la ciencia, tecnología y medio ambiente, con aquellos estudios que integran los principales cambios sufridos en Costa Rica a nivel económico y social desde el siglo XIX, han permitido hasta ahora llevar a cabo nuevas interpretaciones históricas de Costa Rica y ha posibilitado, de manera hasta ahora parcial, la definición y caracterización de los procesos de cambio social en su relación con dichos fenómenos.

La posibilidad de observar a la sociedad costarricense a través del prisma tecnocientífico, y viceversa, la posibilidad de ver a la tecnología y la ciencia con los lentes de la sociedad y el medio ambiente, propone (re)interpretar algunos aspectos clave de los procesos históricos en Costa Rica y Centroamérica desde una perspectiva socio-técno-científica, y además inter, trans y multidisciplinaria. Este esfuerzo actualmente se lleva a cabo a través dentro del programa ACTS, que se presenta como un gran avance en esta ambiciosa agenda. La toma de conciencia de la trayectoria en la producción historiográfica implica, casi por obligación, el compromiso de retomar todos los aprendizajes construidos de forma colectiva en estas últimas décadas. El presente texto es tan sólo un esfuerzo inicial por visibilizar y articular todo este cuerpo de conocimiento. 
Desde luego, el balance deberá ser actualizado al calor de los avances que se construyan tanto en la UNA como en la UCR, en donde se ha observado recientemente una clara aceleración en la producción académica, así como en la formación de profesionales en el campo y en la adaptación de nuevas metodologías y abordajes teóricos. Para señalar dos de los más importantes, destaco las nuevas posibilidades de análisis estadísticos a gran escala en las investigaciones sobre historia agroecológica y ambiental en la UNA, y las posibilidades teóricas derivadas de la confluencia de los estudios CTS y la historia ambiental, lo que desde el programa de investigación ACTS se ha denominado historia tecno-ambiental (Pritchard, 2011).

\section{AGRADECIMIENTOS}

Esta investigación es un producto de la Red Temática de historia de la microbiología en contexto global. Siglos XIX-XXI (B8787) y del proyecto de Historia socio-técnica de la microbiología en Costa Rica en el contexto global. 1870-2015 (B8083), ambos inscritos ante la Vicerrectoría de Investigación de la UCR. Se agradece al CIHAC por su apoyo y estímulo constante a los jóvenes investigadores y se aprecia profundamente la aguda lectura y recomendaciones por parte de los investigadores e investigadoras del programa de investigación en ACTS. Por supuesto, cualquier omisión o error en este texto es de entera responsabilidad del autor.

\section{NOTAS}

1 La Revista de Historia se encuentra digitalizada en el sitio web https://www.revistas.una.ac.cr/ index.php/historia

\section{REFERENCIAS}

Aguilar, J., Barboza, C. \& León, J. (1982). El desarrollo tecnológico del café en Costa Rica y las políticas científicas tecnológicas. San José, Costa Rica: Consejo Nacional de Investigaciones Científicas y Tecnológicas.

Amador, J. (1997). Elliott Coen París (1921-1997): Naturalista y forjador de la meteorología moderna en Costa Rica. Boletín del Instituto Meteorológico Nacional, Marzo, 7-11.

Amador, J. (2002a). Clima y variabilidad climática en Costa Rica a través de información histórica del siglo XIX. Estudios sobre historia y ambiente en América. En García, Bernardo y Prieto, María del Rosario (Comps.), Estudios sobre historia y ambiente en América (pp. 37-54). México: El Colegio de México, IPGH. 
Amador, J. (2002b). Los albores de la física y el desarrollo de la meteorología en Costa Rica. En: Peraldo, Geovanny (Ed.), Ciencia y Técnica en la Costa Rica del Siglo XIX (pp. 187-208). Cartago: Editorial Tecnológica.

Amador, J. y Solano, F. (1991). Algunos datos históricos sobre eclipses de sol en Costa Rica en el Siglo XX. Polaris, 3(2), 9-11.

Amador, J., Páez, J. y Solano, F. (1990). Algunos detalles y hechos históricos de los albores de la Física en Costa Rica: Parte I y II. En Ruiz, Ángel (Ed.), Las matemáticas en Costa Rica.Memorias del Tercer Congreso Nacional de Matemáticas (pp. 349-355). San José: UCR-ITCR.

Arce, A. (1959). Rational Introduction of Technology on a Costa Rican Hacienda: Sociological Implications (Tesis de Doctorado). East Lansing: Michigan State University.

Barrantes, H. y Ruiz, Á. (2000). "La Reforma Liberal y las matemáticas en la Costa Rica del siglo XIX”. Revista Española de Historia de las Ciencias y las Técnicas, 46(23).

Botey, A. (2013). Los actores sociales y la construcción de las políticas de salud del Estado liberal en Costa Rica (1850-1940) (Tesis de Doctorado académico en Historia). San Pedro: Universidad de Costa Rica.

Botey, A. (2019). Los orígenes del Estado del Bienestar en Costa Rica: salud y protección social (18501940). San Pedro: Editorial de la Universidad de Costa Rica.

Calvo, O. y Alfaro, E. (2018). Importancia del alcance en redes del CIGEFI-UCR: Un estudio de los datos, según las publicaciones realizadas en los períodos entre 1979-2015. E-Ciencias De La Información, 8(2), 2-20.

Clare, P. (2011). Los cambios en la cadena de producción de la palma aceitera en el Pacífico costarricense: Una historia económica, socioambiental y tecnocientífica, 1950-2007. San José: Sociedad Editora Alquimia.

Cléves, R. (1995). Tecnología en beneficiado del café. San José: Impresora Tica.

Coto, W. (2015). Las señales del mercado: Revolución Verde y transformaciones socioproductivas en el sistema agroalimentario de la papa en Costa Rica (1950-2010). En Márquez, Esaú y Ortíz, María (Coords), Sociedades encauzadas: geografía, historia y realidad (pp. 265-282). México: Editorial UNICACH/Coneculta.

Coto, W. (2016). La semilla hace la diferencia. Revolución Verde y selección genética en el cultivo de la papa en Costa Rica (1943-2015). Revista Perspectivas Rurales. Nueva Época, 14(28), 88-98.

Díaz, R. (2003). El proceso de institucionalización de la meteorología en Costa Rica (1887-1949) (Tesis de Licenciatura en Historia). San José: Universidad de Costa Rica.

Díaz, R. (2005). Los costarricenses no han dejado de admirar las magnificencias del cielo: La evolución de las ideas astronómicas en Costa Rica (1814-1910). Revista Diálogos, 6(1), 282-307.

Díaz, R. (2007). Exploraciones geográficas e historia natural en Costa Rica: el Instituto FísicoGeográfico Nacional (1889-1903). En Mendoza, Lértora \& Celina, Ana (Eds.),-Geografía 
e historia natural: hacia una historia comparada: estudio a través de Argentina, México, Costa Rica y Paraguay .. Buenos Aires: Fundación para el Estudio del Pensamiento Argentino e Iberoamericano.

Díaz, R. (2009). Estado, comunidades científicas y exploraciones geográficas en Costa Rica: los proyectos cartográficos del Instituto Físico-Geográfico Nacional (1889-1903). En Viales, Ronny, Amador, Jorge, et al. (Eds), Concepciones y representaciones de la naturaleza y la ciencia en América Latina (pp. 211-219). San José: Universidad de Costa Rica

Díaz, R. y Solano,F. (2006). Las ciencias médicas y su aporte a la institucionalización de la meteorología en Costa Rica (1678-1936). Revista Diálogos, 7(1), 150-183.

Enríquez, F. (2001). La función del historiador en los estudios climáticos aportes y perspectiva. Diálogos Revista Electrónica de Historia, 2(3), 1-17.

Fernández, J. (1986). La formación de una hacienda cafetalera en 1845. Un intento de transmisión de tecnología agrícola. Revista de Historia, 14, 199-202.

Fernández, W. (1994). Historia del Desarrollo de las Ciencias Atmosféricas en la Universidad de Costa Rica: Hasta 1995. Ciencia y Tecnología, 18(1-2), 31-46.

Fernández,W.(1995). Desarrollo de la Física en la Universidad de Costa Rica: 1994. Ciencia y Tecnología para América Central: Planes y Estrategias (pp. 127-135). San Salvador: CONACYT.

Fernández, W. (1998). Los Antecedentes de la Creación del Centro de Investigaciones Geofísicas (CIGEFI) de la Universidad de Costa Rica. Revista Geológica de América Central, 21, 99-104.

Fernández, W. y Páez, J. (2000). Las Ciencias del Espacio en Costa Rica. Desarrollo Científico y Tecnológico en Costa Rica: Logros y Perspectivas (pp. 99-110, Tomo II). San José: Academia Nacional de Ciencias.

Fernández, W. (2000). Algunas Ideas para Lograr un Mayor Desarrollo Científico y Tecnológico de Costa Rica". En Desarrollo Científico y Tecnológico en Costa Rica: Logros y Perspectivas (pp. 165-171, Tomo III). San José: Academia Nacional de Ciencias.

Goebel, A. (2008). Historia ambiental, representaciones sociales y exploración decimonónica: elementos conceptuales y empíricos para el estudio del imaginario ambiental de la Costa Rica del siglo XIX. Revista Diálogos, 9(2), 23-53.

Goebel, A. (2009). Una lluvia de males: el régimen de precipitaciones en la Costa Rica del Progreso. Trayectoria, representaciones sociales e impacto socioeconómico (1860-1940). Revista de Historia, 59-60, 57-97.

Goebel, A. (2013). Los bosques del "progreso". Explotación forestal y régimen ambiental en Costa Rica: 1883-1955. San Pedro: Universidad de Costa Rica: Serie Estudios Sociales de la Ciencia, la Técnica y el Medio Ambiente.

Goebel, A. y Viales, R. (2010). Blaming it on the Weather: The role of "inclement" rainfall in Society Nature Relations in Liberal Costa Rica. Global Environment A Journal of History and Natural and Social Sciences, 6, 8-67. 
Gómez, L. y Savage, J. (1991). Investigadores en aquella rica costa: Biología de campo costarricense. 1400-1980. En Janzen, Daniel (Ed.), Historia natural de Costa Rica (pp. 1-11). San José: Editorial de la Universidad de Costa Rica.

Granados, R. (2013). Producción de carbón y leña: un análisis de las actividades productivas relacionadas con el uso del bosque en el cantón de Dota (1950-2007). En Clare, Patricia (Ed.), Teoría y métodos para una historia ambiental costarricense . San José: Editorial Nuevas Perspectivas.

Hall, C. (1978). Cóncavas: formación de una hacienda cafetalera, 1889-1911. San Pedro: Editorial de la Universidad de Costa Rica.

Infante-Amate, J. y Picado, W. (2017). Energy flows in the coffee plantations of Costa Rica: from traditional to modern systems (1935-2010). Regional Environmental Change, 18(4), 1059-1071.

Infante-Amate, J., Picado, W. y Guzmán, G. (2017). Energy Return on Investment in traditional and modern agricultures. Coffee agro-ecosystems in Costa Rica from an agro-ecological perspective (1935-2010) (pp. 157-176). En Gloria Guzmán y Manuel González de Molina (Eds.), Energy in Agroecosystems: A Tool for Assessing Sustainability. New York: Taylor \& Francis/CRC Press.

Infante, J. y Picado, W. (2016, enero). La transición socio-ecológica en el café costarricense. Flujos de energía, materiales y uso del tiempo (1935-2010). Old and New Worlds: The Global Challenges of Rural History. Conferencia Internacional ISCTE-IUL, Lisboa.

Ledezma, R. y Granados, R. (2008). Degradación ambiental y caficultura en Tarrazúentre 1970 y 2006. Revista de Ciencias Ambientales, 36(2), 11-18.

León, J. y Arroyo, N. (2012). Desarrollo histórico del sector agroindustrial de la caña de azúcar en el siglo XX: aspectos económicos, institucionales y tecnológicos. San José: UCR-IICE..

León, J., Arroyo, N. y Montero, A. (2016). La industria en Costa Rica en el siglo XX. San José: EUCR.

Loomis, C., Morales, J., Clifford, R. y Olen, L. (1958). Turrialba: Social Systems and the Introduction of Change. Glencoe, Illinois: Free Press.

López, M. y Granados, R. (2016). Desnudando el mito: un balance sobre las tensiones del modelo de conservación en Costa Rica (1970-2015). Historia Ambiental Latinoamericana y Caribeña (HALAC), 6(1), 61-85.

López,M.\& Picado, W.(2012). Plantas, fertilizantes y transición energética en la caficultura contemporánea de Costa Rica. Bases para una discusión. Revista de Historia, 65-66 (enero), 17-51.

Mendoza, Lértora \& Celina, Ana (2007, 2008, 2009). Geonaturalia: Geografía e Historia Natural: hacia una historia comparada. Estudio a través de Argentina, México, Costa Rica y Paraguay, vol. 1-2. Buenos Aires: Ediciones FEPAI.

Naranjo, C. (1997). La modernización de la caficultura costarricense 1890-1950 (Tesis de Maestría). San Pedro: Universidad de Costa Rica.

Naranjo, C. (2007). Los sistemas de beneficiado del café costarricense: 1830-1914. Revista de Historia, (55-56), 39-72. 
Páez, J. (1994). Aspectos históricos de la astronomía en Costa Rica. Ciencia y tecnología, 18(1-2), 51-73.

Páez, J., Jiménez, M. y Leandro, G. (1995). Historia del Campo Geomagnético de Costa Rica. Ciencia y tecnología, 19(1-2), 53-78.

Peraldo, G. (2002). Ciencia y Técnica en la Costa Rica del Siglo XIX. Cartago: Editorial Tecnológica.

Peraldo, G. y Amador, J. (2007). Las raíces filosóficas y físicas de las hipótesis sobre sismología desarrolladas por el Ing. Luis Matamoros. Una influencia de las corrientes Aristotélicas y Feijoosistas a inicios del siglo XX. Revista Geológica de América Central, 37, 65-77.

Peraldo, G., Solano, F. y Quesada, A. (2009). La plaga de langosta en Costa Rica entre 1850 y 1950. En: Mendoza, Lértora y Celina (Eds.), Ana. Geonaturalia: Geografía e Historia Natural: hacia una historia comparada. Estudio a través de Argentina, México, Costa Rica y Paraguay (pp. 139-184.). Buenos Aires: Ediciones FEPAI.

Picado, W. (2000). La expansión del café y el cambio tecnológico desigual en la agricultura del cantón de Tarrazú (Tesis de Maestría en Historia Aplicada con énfasis en Estudios Agrarios). Heredia: Universidad Nacional Costa Rica.

Picado, W. (2011). Breve historia semántica de la Revolución Verde(pp. 25-50). En Lanero, Daniel y Dulce Freire (Eds.), Agriculturas e innovación tecnológica en la Península Ibérica (19461986) (pp. 25-50). España: Ministerio de Medio Ambiente, Medio Rural y Marino.

Picado, W. (2012). En busca de la genética guerrera. Segunda Guerra Mundial, cooperación agrícola y Revolución Verde en la agricultura de Costa Rica. Revista de Historia Agraria, 56, 107-134.

Picado, W. (2013). El regreso de la Cornucopia. El debate sobre la primera y segunda Revolución Verde. Avances en Seguridad Alimentaria y Nutricional, 5(1), 1-18.

Picado, W., Ledezma, R. y Granados, R. (2009). Territorio de coyotes, agroecosistemas y cambio tecnológico en una región cafetalera de Costa Rica. Revista de Historia, 59-60, 119-165.

Pizarro, Y. (2016a). Diques y dragas para el conflicto: recurso hídrico, modernización agropecuaria y la exclusión del modelo comunal campesino (cuenca media del río Tempisque) en perspectiva histórica. HALAC, 6(1), 30-60.

Pizarro, Y. (2016b). Impacto de las inundaciones en la modernización productiva de la llanura aluvial del río Tempisque (1950-1975)(pp. 155-161). En Zúñiga, Y. (Ed), Perspectivas interdisciplinarias: riesgo y vulnerabilidad ante fenómenos hidrometeorológicos (pp. 155-161). Heredia: EUNA.

Pritchard, S. (2011). Confluence: The Nature of Technology and the Remaking of the Rhône.Cambridge, Mass./London: Harvard University Press.

Rojas, G. (2000). Café, ambiente y sociedad en la cuenca del río Virilla, Costa Rica (1840-1955). San José: Editorial de la Universidad de Costa Rica.

Rojas, M., Samper, M. y Torres, M. (1994). Cambio tecnológico, ciclo laboral y productividad del trabajo en una empresa cafetalera costarricense, 1946-1961. Revista de Historia, 30, 103-136. 
Ruiz, Á. (2001). Ideologías y extranjeros en la educación y las matemáticas de Costa Rica durante el siglo XIX. Llull: Revista de la Sociedad Española de Historia de las Ciencias y de las Técnicas, 23(48), 661-688.

Ruiz, Á. (Ed.). (1990). Las matemáticas en Costa Rica. Memorias del Tercer Congreso Nacional de Matemáticas. San José: UCR-ITCR.

Ruiz, Á. (Ed.). (1995). Historia de las matemáticas en Costa Rica. San José: EUCR y EUNA.

Ruiz, Á. y Rodríguez, P. (2003). Educación y matemáticas en la Universidad de Santo

Tomás (s.p.). En Peraldo, Giovanni (Ed.), Historia de las ciencias y las técnicas en la Costa Rica del siglo XIX. Cartago: Editorial Tecnológica de Costa Rica. Recuperado de shorturl.at/elwAR

Samper, M. (1993). Policultivo, modernización y crisis: paradojas del cambio técnico/social en la caficultura centroamericana. Revista de Historia, 27, 111-145.

Samper, M. (1994). Modelos vrs. Prácticas. Acercamiento inicial a la cuestión tecnológica en algunos manuales sobre caficultura, 1774-1895. Revista de Historia, 30, 11-40.

Samper, M. (2001). Construcción histórica de la calidad y la competitividad. En Samper, Mario y Sfez, Paul (Eds.). La cadena de producción y comercialización del café: perspectiva histórica y comparada. San José: Progreso editorial.

Samper, M., Naranjo, C. y Sfez, P. (2000). Entre la tradición y el cambio: Evolución tecnológica de la caficultura costarricense. Heredia: Universidad Nacional e Instituto Panamericano de Geografía e Historia.

Sfez, P. (2000). Revolución Verde y cambio tecnológico en la caficultura(pp. 57-72). En Peters, Gertrud (Ed.), Entre la tradición y el cambio: Evolución tecnológica de la caficultura costarricense (pp. 57-72). San José: SEE S.A.

Solano, F. y Amador, J. (1994). La historia de la física en Costa Rica: Ing Henry Mcghie Boyd. Ciencia y Tecnología, 18(1-2), 9-19.

Solano, F. y Amador, J. (1991). Algunos detalles de eclipses de sol en Costa Rica en el Siglo XIX. Acodea, 3(2), 3-7.

Solano, F. y Páez, J. (1990). Semblanza de un Profesor de Ciencias: Don Elliott Coen París. En Ruiz, Ángel (Ed.), Ciencia y Tecnología en la construcción del futuro . San José: Editorial Guayacán.

Vargas Mena, E. (2000). La Educación Ambiental y la Educación Indígena en Costa Rica Perspectiva histórica y opciones actuales. San José: UICN, UNA, UNED, MINAE.

Vargas Mena, E. (2001). Problemas urgentes de la educación ambiental: el caso de la tortuga verde (Chelonia mydas) en el Caribe costarricense. Tópicos en Educación Ambiental, 3(7), 9-21. 
Vargas Trejos, Y. (2015). Exposición a agroquímicos y creencias asociadas a su uso en la cuenca hidrográfica del Río Morote, Guanacaste, Costa Rica: Un estudio de casos. Ciencia \& Trabajo, 17(52), 54-68.

Viales, R. (2003). Antecedentes de los estudios sociales de la ciencia, la técnica y el ambiente en la Universidad de Costa Rica. San José: Centro de Investigaciones Geofísicas de la Universidad de Costa Rica.

Viales, R. (Ed.). (2010). El contexto, los problemas y los actores de la definición de políticas científicas para la cohesión social en América Latina: Una visión desde Costa Rica. San José: Sociedad Editora Alquimia.

Viales, R. y Montero, A. (s.f). Los inicios frustrados de la mecanización de la agricultura costarricense entre 1900 y 1950. La difusión del arado y del tractor. Actores, tecnología agrícola, discursos y representaciones desde una perspectiva transnacional. En Viales, Ronny \& Granados, Rafael (Eds.), Trayectoria y dinámica del sector agrario-rural costarricense en el contexto global. 1850-2018. En prensa, Editorial Sede del Pacífico, Universidad de Costa Rica.

Viales, R., Amador, J. y Solano, F. (Eds.). (2009). Concepciones y representaciones de la naturaleza y la ciencia en América Latina. San Pedro: Vicerrectoría de Investigación, Universidad de Costa Rica.

Vicerrectoría de Investigación. (1997). Meteorología e impacto social regional: Centro América y México (MISCAM). Universidad de Costa Rica, Sistema de Información y Gestión de Proyectos, Programas y Actividades (SIGPRO). Recuperado de https://vinv.ucr.ac.cr/sigpro/ web/projects/97519

Vicerrectoría de Investigación. (2010). Tierra, aire, agua y bosques. Historia del medio ambiente en la Costa Rica de los siglos XIX y XX. Universidad de Costa Rica, Sistema de Información y Gestión de Proyectos, Programas y Actividades (SIGPRO). Recuperado de https://vinv.ucr. ac.cr/sigpro/web/projects/B0652

Vicerrectoría de Investigación. (2016). Ambiente, ciencia, tecnología y sociedad (ACTS). Intersección Entre historia ambiental y estudios sociales de la ciencia, la tecnología y la sociedad (CTS). Universidad de Costa Rica, Sistema de Información y Gestión de Proyectos, Programas y Actividades (SIGPRO). Recuperado de https://vinv.ucr.ac.cr/sigpro/web/projects/B6901

Vicerrectoría de Investigación. (2018). Red: historia de la microbiología en contexto global. Siglos XIXXXI. Universidad de Costa Rica, Sistema de Información y Gestión de Proyectos, Programas y Actividades (SIGPRO). Recuperado de https://vinv.ucr.ac.cr/sigpro/web/projects/B8787 